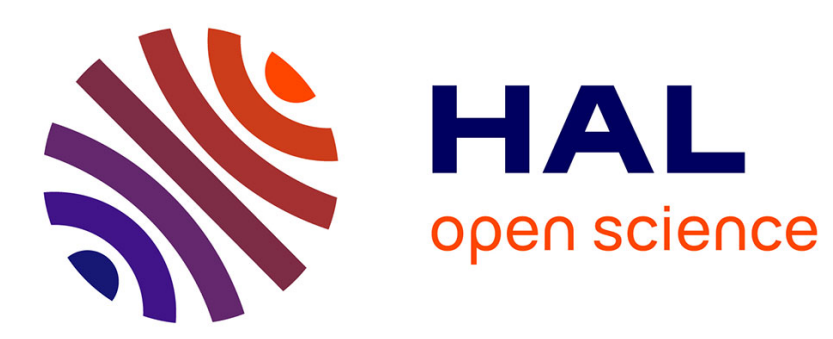

\title{
On the MAX MIN VERTEX COVER problem
}

Nicolas Boria, Federico Della Croce, Vangelis Paschos

\section{To cite this version:}

Nicolas Boria, Federico Della Croce, Vangelis Paschos. On the MAX MIN VERTEX COVER problem. 2013. hal-00874216

\section{HAL Id: hal-00874216 https://hal.science/hal-00874216}

Preprint submitted on 17 Oct 2013

HAL is a multi-disciplinary open access archive for the deposit and dissemination of scientific research documents, whether they are published or not. The documents may come from teaching and research institutions in France or abroad, or from public or private research centers.
L'archive ouverte pluridisciplinaire HAL, est destinée au dépôt et à la diffusion de documents scientifiques de niveau recherche, publiés ou non, émanant des établissements d'enseignement et de recherche français ou étrangers, des laboratoires publics ou privés. 


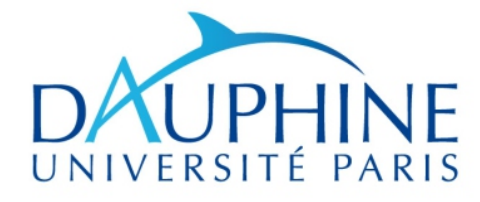

Laboratoire d'Analyses et Modélisation de Systèmes pour l'Aide à la Décision UMR 7243

\section{CAHIER DU LAMSADE 343}

Octobre 2013

On the MAX MIN VERTEX COVER problem

N. Boria, F. Della Croce, V.Th. Paschos

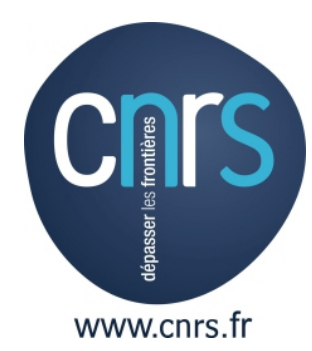




\title{
On the MAX MIN VERTEX COVER problem*
}

\author{
Nicolas Boria ${ }^{1}$, Federico Della Croce $^{1}$, and Vangelis Th. Paschos ${ }^{2,3}$ \\ ${ }^{1}$ Politecnico di Torino, DAUIN \\ \{nicolas.boria, federico.dellacroce\}@polito.it \\ ${ }^{2}$ PSL Research University, Université Paris-Dauphine, LAMSADE \\ CNRS UMR 7243 paschos@lamsade.dauphine.fr \\ ${ }^{3}$ Institut Universitaire de France
}

October 2, 2013

\begin{abstract}
We address the MAX MIN VERTEX COVER problem, which is the maximization version of the well studied MIN INDEPENDENT DOMINATING SET problem, known to be NP-hard and highly inapproximable in polynomial time. We present tight approximation results for this problem on general graphs, namely a polynomial approximation algorithm which guarantees an $n^{-1 / 2}$ approximation ratio, while showing that unless $\mathbf{P}=\mathbf{N P}$, the problem is inapproximable within ratio $n^{\varepsilon-(1 / 2)}$ for any strictly positive $\varepsilon$. We also analyze the problem on various restricted classes of graph, on which we show polynomiality or constant-approximability of the problem. Finally, we show that the problem is fixed-parameter tractable with respect to the size of an optimal solution, to tree-width and to the size of a maximum matching.
\end{abstract}

\section{Introduction}

In the MIN INDEPENDENT DOMINATING SET problem, also called MIN INDEPENDENT DOMINATING SET, given a graph $G(V, E)$, we are asked to determine a minimum size vertex-subset that is simultaneously independent and dominating. This problem has been proved to be inapproximable within $n^{1-\varepsilon}$, for any $\epsilon>0$, not only in general graphs [15] but also in restricted graph classes as, for instance, the circle graphs [7]. Also, and probably due to this fact, exact solution of MIN INDEPENDENT DOMINATING SET in general or in restricted classes of graphs by moderately exponential algorithms has received a growing attention

${ }^{*}$ Research supported by the French Agency for Research under the program TODO, ANR09-EMER-010 and by a Lagrange fellowship of the Fondazione CRT, Torino, Italy 
in the past years $[14,13,4]$. This problem has also been tackled using exponential approximation techniques [4]. Finally, it is shown to be very hard from a parameterized complexity point of view since it is $\mathbf{W}[2]$-hard [8].

Surprisingly, to the best of our knowledge, the natural symmetric problem, the MAX MIN VERTEX COVER problem, where the goal is to compute a minimal (for exclusion) vertex cover of maximum size, has not been addressed yet. This problem obviously has the same characteristics as its minimization counterpart in terms of NP-hardness and exact computation, but might have different behaviours in terms of approximability and parameterized complexity (as in the case of the pair MAX INDEPENDENT SET - MIN VERTEX COVER). Indeed, this seems in a large extent to be the case.

We show in this paper that, while also highly inapproximable, MAX MIN VERTEX COVER is better approximable than its mate, since it can be approximately solved in polynomial time within ratio $n^{1 / 2}$, where $n$ is the size of the input graph. This result is matched by an inapproximability bound of $n^{\varepsilon-(1 / 2)}$ that can be extended also to an $O(1 / \Delta)$ inapproximability bound, where $\Delta$ is the maximum degree of the input graph. We also match it to an $O(3 / 2 \Delta)$ approximation ratio achieved by a natural greedy algorithm. We also prove that, unlike MIN INDEPENDENT DOMINATING SET, MAX MIN VERTEX COVER is in FPT, the class of fixed-parameter tractable problems not only with respect to the standard parameter, i.e., the value of the optimum, but also with respect to the cardinality of a maximum matching (that is smaller than the value of the optimum). Let us note that both MIN WEIGHTED DOMINATING SET and MAX WEIGHTED INDEPENDENT SET are polynomially solvable in graphs with bounded treewidth $[1,2,3]$ (and, actually, fixed parameter tractable with respect to the treewidth of the input graph [19]). With similar dynamic programming techniques, it can be shown that also both WEIGHTED MAX MIN VERTEX COVER and WEIGHTED MIN INDEPENDENT DOMINATING SET are fixed parameter tractable with respect to the treewidth. Since the techniques used for obtaining this result are quite similar to those in [19], the proof of the result is omitted. We conclude the paper by studying complexity of MAX MIN VERTEX COVER in interval graphs.

\section{Approximation of MAX MIN VERTEX COVER in general graphs}

We give in this section inapproximability upper bounds matched by lower bounds achieved in polynomial time for MAX MIN VERTEX COVER. We first study ratios functions of $n$ and then functions of $\Delta$.

Proposition 1. For any positive constant $\varepsilon$, MAX MIN VERTEX COVER is inapproximable within ratio $O\left(n^{\varepsilon-(1 / 2)}\right)$ unless $\mathbf{P}=\mathbf{N P}$.

Proof. First, recall that MAX IndEPENDENT SET has been proved to be inapproximable within ratio $n^{\varepsilon-1}$ for a given $\varepsilon \in(0,1)[16]$ unless $\mathbf{P}=\mathbf{N P}$. Suppose that there exists an $\varepsilon$-approximation algorithm A for MAX MIN VERTEX COVER. 
Consider an unweighted instance of MAX INDEPENDENT SET given by a graph $G(V, E)$. Out of this instance of MAX INDEPENDENT SET, we build an instance $H\left(V \cup S, E^{\prime}\right)$ of MAX MIN VERTEX COVER in the following way: for each vertex $v$ of $V$ one adds $n+1$ vertices connected only to $v$ in $H$, while the inner edges of the set $V$ are left unchanged. In other words, graph $H$ is obtained by adding an independent set $S$, of order $n^{2}+n$ to the initial graph $G$, and connecting $n+1$ vertices of the independent set to each vertex $v$.

Note that the graph $H$ can be built in polynomial time, and has precisely $n^{2}+2 n$ vertices. Denote by $\operatorname{opt}(G)$ an optimal independent set in $G$, and by $\operatorname{opt}(H)$ an optimal vertex cover in $H$.

Consider a vertex cover $\operatorname{SOL}(H)$ that has value $\operatorname{sol}(H)$ in $H$. First, notice that $H$ admits a maximal matching of $n$ edges, that consists of taking, for each vertex of $V$, one edge linking this vertex to one of its neighbors in $S$. Hence, any vertex cover in $H$ takes at least $n$ vertices, i.e., $\operatorname{sol}(H) \geqslant n$

Notice also that, for any vertex $v$ of $V$ that does not belong to $\mathrm{SOL}(H)$, then $\operatorname{SOL}(H)$ must take all its neighbors in $S$, that is $n+1$ vertices. Moreover the set $V \backslash \operatorname{SOL}(H)$ of vertices of $V$ that do not belong to $\operatorname{SOL}(H)$ defines an independent set in $G$ of weight $n-\operatorname{sol}(H)$. In other words, one can assert that any solution $\operatorname{SOL}(H)$ of value $\operatorname{sol}(H)$ in $H$ can be easily transformed into an independent set $\operatorname{SOL}(G)$ in $G$ of value:

$$
\operatorname{sol}(G) \geqslant \frac{\operatorname{sol}(H)-n}{n}
$$

Conversely, the existence of a maximal independent set of size $h$ in $G$ induces the existence of a minimal vertex cover of size $n h+n$ in $H$. It suffices to consider the following vertex cover: all vertices of $V$ that do not belong in the independent set $(n-h$ vertices), and all vertices of $S$ linked to a vertex of the independent set $(h(n-1)$ vertices $)$. Therefore, it holds that a minimal vertex cover of size $n \cdot \operatorname{opt}(G)+n$ exists in $H$. In other words, opt $(H) \geqslant n \cdot \operatorname{opt}(G)+n$.

Now, for some constant positive $\rho<1$, suppose that there exists a polynomial time algorithm A for MAX MIN VERTEX COVER that guarantees an approximation ratio $n^{-\rho}$, and suppose that a solution $\operatorname{SOL}(H)$ has been computed by this algorithm on graph $H$. Reminding that graph $H$ has $O\left(n^{2}\right)$ vertices, the approximation ratio guaranteed by $\mathrm{A}$ on $H$ turns to be $n^{-2 \rho}$. Then, it holds that:

$$
\operatorname{sol}(H) \geqslant n^{-2 \rho} \cdot \operatorname{opt}(H)
$$

By combining (1) and (2), one can assert that $\operatorname{SOL}(H)$ can easily be transformed into an independent set $\operatorname{SOL}(G)$ in $G$ of value:

$$
\begin{aligned}
\operatorname{sol}(G) & \geqslant \frac{n^{-2 \rho} \cdot \operatorname{opt}(H)-n}{n} \geqslant n^{-2 \rho-1} \cdot \operatorname{opt}(H)-1 \\
& \geqslant n^{-2 \rho} \cdot \operatorname{opt}(G)+n^{-2 \rho}-1 \geqslant n^{-2 \rho} \cdot \operatorname{opt}(G)
\end{aligned}
$$

where the last inequality holds for $n$ big enough.

Hence, the existence of an $n^{-\rho}$-approximation algorithm A for MAX MIN VERTEX COVER induces the existence of an $n^{-2 \rho}$-approximation algorithm for 
MAX INDEPENDENT SET, that would consist of:

- building the instance $H$ of MAX MIN VERTEX COVER out of the instance $G$ of MAX INDEPENDENT SET ;

- running the algorithm A on the instance $H$ that outputs a solution $\operatorname{SOL}(H)$;

- returning the solution $V \backslash \operatorname{SOL}(H)$ for the initial problem.

Since, for any constant $0<\varepsilon \leqslant 1$, the existence of an $n^{1-\varepsilon}$-approximation algorithm for MAX WEIGHTED INDEPENDENT SET induces $\mathbf{P}=\mathbf{N P}[16]$, an $n^{-\rho_{-}}$ approximation algorithm for MAX MIN VERTEX COVER can exist only subject to the condition that $n^{-2 \rho} \leqslant n^{1-\varepsilon}$ for any $0<\varepsilon \leqslant 1$. This leads to $\rho \leqslant \varepsilon-1 / 2$, which concludes the proof.

Observe now that the order of graph $H$ in the gap-reduction of Proposition 1 is $O\left(n^{2}\right)$, while the maximum degree of $H$ is $O(n)$. Then, the following inapproximability bound also can be immediately derived.

Corollary 1. MAX MIN VERTEX COVER is inapproximable in polynomial time within ratios $O\left(\Delta^{\epsilon-1}\right)$, for any $\epsilon>0$.

Let us now recall the following very classical and obvious observation that will be used later.

Remark 1. Denoting by $M$ a maximum matching of a graph $G$, any vertex cover (a fortiori a minimal one) of $G$ uses more than $|M|$ vertices (since, at least one distinct vertex is needed per one edge of $M$ ).

Lemma 1. Consider a graph $G(V, E)$ and an independent set $S$ of $G$. Denote by $\Gamma(S)$ the set of neighbors of $S$, and $V^{\prime}=V \backslash(S \cup \Gamma(S))$. Finally, denote by $\operatorname{SOL}\left(G^{\prime}\right)$ a minimal vertex cover on the induced subgraph $G\left[V^{\prime}\right]$ and by $\operatorname{sol}\left(G^{\prime}\right)$ its cardinality. It holds that $\Gamma(S) \cup S O L\left(G^{\prime}\right)$ is a feasible solution for MAX MIN VERTEX COVER.

Proof. First, let us prove that $\Gamma(S) \cup \operatorname{sol}\left(V^{\prime}\right)$ is a vertex cover: all edges of $S \times \Gamma(S)$ and $\Gamma(S) \times V^{\prime}$ are covered by vertices of $\Gamma(S)$, and all edges inside the induced subgraph $G\left[V^{\prime}\right]$ are covered by $\operatorname{SOL}\left(G^{\prime}\right)$. By hypothesis, $S \times S=\emptyset$, so that $\Gamma(S) \cup \operatorname{SOL}\left(G^{\prime}\right)$ is, indeed, a vertex cover.

Then, let us establish the minimality of such a vertex cover: on the one hand, no vertex of $\Gamma(S)$ can be removed, as they all cover an edge linked to a vertex of $S$ (and no vertex of $S$ is in the vertex cover), and on the other hand, $\operatorname{SOL}\left(G^{\prime}\right)$ is a minimal vertex cover on a subgraph of $G$, so that none of its vertices can be removed without uncovering an edge.

Proposition 2. MAX MIN VERTEX COVER is approximable within ratio $n^{-1 / 2}$ in polynomial time.

Proof. Consider a graph $G(V, E)$, with $|V|=n$. Let $\Gamma(x)$ be the set of neighbors of a given vertex $x$ and, given $V^{\prime} \subseteq V$, let $G\left[V^{\prime}\right]$ be the subgraph of $G$ induced by the set of vertices $V^{\prime}$. Consider the following approximation algorithm for MAX MIN VERTEX COVER:

- compute a maximum matching $M$; 
- among the matched vertices, let $x$ be the one with the maximal number of exposed neighbors;

- compute a minimal vertex cover on $G\left[V^{\prime}\right]$ with a greedy algorithm, where $V^{\prime}=V \backslash(\{x\} \cup \Gamma(x))$, and denote it by $\operatorname{SOL}\left(G^{\prime}\right)$;

- output $\operatorname{SOL}(G)=\Gamma(x) \cup \operatorname{SOL}\left(G^{\prime}\right)$.

First, by Lemma 1, we can assert that the solution returned by our approximation algorithm is feasible. Then notice that the algorithm runs in polynomial time, all steps of the algorithm are so: step 1 can be performed in $O\left(n^{2.376}\right)$ by the algorithm presented in [17], identifying vertex $x$ is done in $O(n)$, and building a minimal vertex cover is done in $O\left(n^{2}\right)$ (starting from the whole set of vertices, the greedy algorithm deletes them one by one as long as the solution remains a vertex cover, when no vertex can be deleted, the remaining set is a minimal vertex cover). Finally, without loss of generality, let us suppose that the graph has no isolated vertices, since such vertices obviously make the problem easier to approximate.

Let us now analyze the approximation guarantee of this algorithm. Given the maximum matching $M$ computed at the first step of the algorithm, denote by $P$ the set of exposed vertices of $V$ with respect to $M$ (i.e., the set of vertices of $V$ that are not saturated by $M$ ), which obviously forms an independent set. Finally, set $p=|P|$ and $m=|M|$. Our analysis is based upon the following maximality argument that will be used also in Proposition 3.

Notice that each edge $\left(v_{i}, v_{j}\right)$ of $M$ is linked to a set of vertices $P_{i j} \subseteq P$, so that $P_{i j} \subset \Gamma\left(v_{i}\right)$, or $P_{i j} \subset \Gamma\left(v_{j}\right)$. Indeed, suppose $v_{i}$ has some neighbor $v_{k}$ in $P$ not linked to $v_{j}$, and $v_{j}$ some neighbor $v_{l}$ in $P$ not linked to $v_{i}$. Then, by deleting $\left(v_{i}, v_{j}\right)$ from $M$ and adding $\left(v_{i}, v_{k}\right)$ and $\left(v_{j}, v_{l}\right)$ to it, one could produce a matching with $m+1$ edges, so that $M$ would not be a maximum matching. In other words, there exists a covering of $P$ by $m$ sets $P_{i j}$, each of them been included in the neighborhood of a single matched vertex. For the algorithm, this implies that the vertex $x$ picked at step 2 has at least $p / m$ neighbors in $P$.

The algorithm includes the whole neighborhood of this vertex $x$, which might also include some matched vertices. Suppose that $\Gamma(x)$ contains exactly $h$ matched vertices. Then it holds that:

$$
|\Gamma(x)| \geqslant h+\frac{p}{m}=h+\frac{n-2 m}{m}
$$

We now bound the value of $\operatorname{SOL}\left(G^{\prime}\right)$. Among the edges of the matching $M$, at least $m-h$ still exist in the subgraph $G\left[V^{\prime}\right]$. Indeed, this subgraph is obtained by deleting from $G$ the vertex $x$ together with all its neighbors, and all edges incident to these vertices. It is clear that, by deleting $h$ vertices from $M$, only $h$ edges are deleted from it. Thus, $G\left[V^{\prime}\right]$ contains a matching with $m-h$ edges, so that any vertex cover in $G\left[V^{\prime}\right]$ has at least $m-h$ vertices. A fortiori, this also holds for the vertex cover computed at step 3 of the algorithm; so:

$$
\operatorname{sol}\left(G^{\prime}\right) \mid \geqslant m-h
$$

Combining (3) and (4), we finally get the following bound on the value of the solution computed by the algorithm: $\operatorname{sol}(G) \geqslant m+\frac{n}{m}-2 \geqslant \sqrt{n}$, where the last 
inequality results from a simple case analysis on the value of $m$ with respect to $\sqrt{n}$ : if $m \geqslant \sqrt{n}$, then the first term of the sum is at least $\sqrt{n}$ and the second at least 2 (as $m \leqslant n / 2)$. In the opposite case $(m<\sqrt{n})$, the second term of the sum is at least $\sqrt{n}$, where $m \geqslant 2$ (if $m=1$, the graph is a star, and the problem is polynomial).

Considering that $\operatorname{opt}(G) \leqslant n$, the algorithm clearly guarantees an $n^{-1 / 2}$ approximation ratio, and the proof is concluded.

Proposition 3. MAX MIN VERTEX COVER is polynomially approximable within ratio $3 / 2 \Delta$, where $\Delta$ is the maximum degree of the input graph. Furthermore, in bounded-degree graphs, regular graphs and graphs admitting a perfect matching MAX Min VERTEX COVER is in $\boldsymbol{A P} \boldsymbol{X}$.

Proof. Denote by $d_{i}$ the degree of a vertex $v_{i} \in V$, by $d$ the average degree of $G$ and, as previously, by $M$ a maximum matching of $G$, by $m$ the cardinality of $M$ and by $p$ the cardinality of the set $P=V \backslash V(M)$ of the exposed vertices of $V$ with respect to $M$.

The maximality argument stated in the proof of Proposition 2, has the following consequence for sets $M$ and $P$ :

for an edge $\left(v_{i}, v_{j}\right) \in M$, if one, say $v_{i}$, of its endpoints has more than one exposed neighbor, then $v_{j}$ has no exposed neighbour at all; in the opposite case, an augmenting path would occur; in other words, in the case that a matching edge $\left(v_{i}, v_{j}\right)$ is incident to some edge $(v, u)$ with either $v_{i}=v$, or $v_{j}=v$, and $u \in P$, it holds that $\left|\left(\Gamma\left(v_{i}\right) \cup \Gamma\left(v_{j}\right)\right) \cap P\right| \leqslant 1$.

Suppose that there exist a set $M^{\prime}$ of $m^{\prime}$ edges of $M$, whose one endpoint is adjacent to some exposed vertex of $G$ with respect to $M$. Obviously, $p \leqslant m^{\prime} \cdot \Delta$. Since $n=2 m+p$, we get, using the quoted consequence above, $n=2 m+p \leqslant$ $2\left(m-m^{\prime}\right)+(\Delta+1) m^{\prime}=2 m+(\Delta-1) m^{\prime} \leqslant(\Delta+1) m$; hence:

$$
m \geqslant \frac{n}{\Delta+1}
$$

By the seminal Turán's Theorem, every maximal (for inclusion) independent set of $G$ has size at least $n /(d+1)$; consequently:

$$
\operatorname{opt}(G) \leqslant \frac{d n}{d+1}
$$

Combining (5) and (6) and taking into account Remark 1, the following holds for the approximation of every minimal vertex cover:

$$
\frac{\operatorname{sol}(G)}{\operatorname{opt}(G)} \geqslant \frac{d+1}{d(\Delta+1)} \simeq \frac{d+1}{d \Delta}
$$

for arbitrarily large values of $\Delta$. Also the following fact holds for any vertex cover of a graph $G(V, E)$ of order $n$. 
Any vertex cover (a fortiori a minimal one) $C$ guarantees approximation ratio at least $(d+1) / 2 \Delta$ for MAX MIN VERTEX COVER.

In fact, since $C$ covers $E$, it holds that $\sum_{v_{i} \in C} d_{i} \geqslant|E|$. Also, $\sum_{v_{i} \in C} d_{i} \leqslant \Delta|C|$ and $|E|=n d / 2$. Putting all this together, we get:

$$
\operatorname{sol}(G)=|C| \geqslant \frac{n d}{2 \Delta}
$$

Combining (8) and (6), we derive:

$$
\frac{\operatorname{sol}(G)}{\operatorname{opt}(G)} \geqslant \frac{d+1}{2 \Delta}
$$

Ratio in (9) is increasing with $d$, while in (7) is decreasing with $d$. Equality holds for $d=2$, which derives ratio $3 / 2 \Delta$.

\section{Parameterized analysis}

We prove in this section that, continuing the asymmetry between MIN INDEPENDENT DOMINATING SET and MAX MIN VERTEX COVER, the later is fixed parameter tractable when parameterized by the standard parameter, i.e., the cardinality opt of a maximum minimal vertex cover.

Proposition 4. MAX MIN VERTEX COVER can be solved in $O^{*}\left(4^{\mathrm{opt} / 3}\right)=$ $O^{*}\left(1.5874^{\mathrm{opt}}\right)$.

Proof. Let, as previously, $\Gamma\left(v_{i}\right)$ be the neighborhood of vertex $v_{i}$. First, notice that if all vertices have degree $\leqslant 2$, then the problem becomes straightforwardly polynomially solvable by dynamic programming. Then, we assume that there exists a vertex $v_{j}$ such that $d_{j} \geqslant 3$. Notice also that for each vertex $v_{i}$ at least one vertex among the set $\Gamma\left(v_{i}\right) \cup\left\{v_{i}\right\}$ cannot be part of the vertex cover or else that vertex cover would not be minimal. We consider a branch and reduce approach where in each branch a vertex is excluded from the vertex cover and its neighbors are then necessarily included. We point out that such branch guarantees that all vertex covers generated will be minimal. We branch on vertex $v_{j}$ according to the following exhaustive cases.

Case 1. $d_{j} \geqslant 3$ and all $v_{i} \in \Gamma\left(v_{j}\right)$ have degree $d_{i} \geqslant d_{j}$. We generate $\left|\Gamma\left(v_{j}\right)\right|+1$ branches as follows: in one branch, vertex $v_{j}$ is excluded from the vertex cover and correspondingly all its neighbors are included; in all other branches one of the vertices $v_{i} \in \Gamma\left(v_{j}\right)$ is excluded while all $v_{k} \in \Gamma\left(v_{i}\right)$ are included. This corresponds to $\left|d_{j}+1\right|$ branches where in each branch at least $\left|d_{i}\right|$ vertices are included in the vertex cover. The worst-case occurs for $\left|d_{j}\right|=$ 3 , where we have four branches each including 3 vertices in the vertex set. Correspondingly, the complexity is $O^{*}\left(4^{\mathrm{opt} / 3}\right)=O^{*}\left(1.5874^{\mathrm{opt}}\right)$.

Case 2. $d_{j} \geqslant 3$ and there exists $v_{i} \in \Gamma\left(v_{j}\right)$ with $d_{i}=2$. Three subcases occur with respect to the degree of the other neighbor $v_{k}$ of $v_{i}$. 
Subcase $2(a)$. If $d_{k} \geqslant 3$, then either $v_{i}$ or $v_{j}$ or $v_{k}$ are excluded from the vertex cover and correspondingly their neighbors are included in the vertex cover. Then, the recursion is at least $T$ (opt) $\leqslant T$ (opt -2$)+2 T($ opt -3$)$ and the worst-case complexity is $O^{*}\left(1.5214^{\mathrm{opt}}\right)$.

Subcase $2(b)$. If $d_{k}=2, v_{j}$ may or may not be adiacent to $v_{k}$. If $v_{j}$ and $v_{k}$ are adjacent, then a branch on $v_{j}$ can be performed: either $v_{j}$ is excluded from the vertex cover and correspondingly its neighbors (at least three) are included in the vertex cover, or $v_{j}$ is included in the vertex cover and arbitrarily $v_{i}\left(v_{k}\right)$ is excluded from the vertex cover and $v_{k}\left(v_{i}\right)$ is included in the vertex cover. Then, the recursion is at least $T(\mathrm{opt}) \leqslant T$ (opt -2$)+T$ (opt -3$)$ and the worstcase complexity is $O^{*}\left(1.3248^{\mathrm{opt}}\right)$. Alternatively, $v_{j}$ and $v_{k}$ are non adjacent and $v_{k}$ is adjacent to another vertex $v_{l}$. Then, either $v_{i}$ is excluded from the vertex cover (and its two neighbors are included), or $v_{k}$ is excluded from the vertex cover (and again its two neighbors are included), or both $v_{i}$ and $v_{k}$ are included in the vertex cover and correspondingly $v_{j}$ and $v_{l}$ are excluded from the vertex cover. In this last case, the other neighbors of $v_{j}$ and $v_{l}$ (that may possibly coincide) must be included in the vertex cover and globally at least four vertices must be included in the vertex cover. Correspondingly, the recursion is at least $T(\mathrm{opt}) \leqslant 2 T$ (opt -2$)+T$ (opt -4 ) and the worst-case complexity is $O^{*}\left(1.5538^{\mathrm{opt}}\right)$ (notice that this last branch does not even occur if $v_{j}$ and $v_{l}$ are adjacent).

Subcase 2 (c). If $d_{k}=1$, then $v_{i}$ and $v_{j}$ cannot be both included in the vertex cover as such solution is not better than the one with $v_{j}$ and $v_{k}$ included in the vertex cover and $v_{i}$ excluded from the vertex cover. Then either $v_{i}$ is excluded from the vertex and two vertices $\left(v_{i}\right.$ and $\left.v_{j}\right)$ are included in the vertex cover, or $v_{j}$ is excluded from the vertex cover and all its neighbors (at least three vertices) are included in the vertex cover. Correspondingly, the recursion is at least $T$ (opt) $\leqslant T($ opt -2$)+T$ (opt -3$)$ and the worst-case complexity is $O^{*}\left(1.3248^{\mathrm{opt}}\right)$.

Case 3. $d_{j} \geqslant 3$ and there exists $v_{i} \in \Gamma\left(v_{j}\right)$ with $d_{i}=1$. We generate 2 branches where either $v_{i}$ is excluded from and $v_{j}$ is included, or $v_{j}$ is excluded from the vertex and all its neighbors are included. Correspondingly, the recursion is at least $T(\mathrm{opt}) \leqslant T(\mathrm{opt}-1)+T(\mathrm{opt}-3)$ and the worst-case complexity is $O^{*}\left(1.4656^{\mathrm{opt}}\right)$.

Overall, the worst-case is attained in case 1 with complexity $O^{*}\left(4^{\text {opt } / 3}\right)=$ $O^{*}\left(1.5874^{\mathrm{opt}}\right)$.

In what follows, we further strengthen the result of Proposition 4, showing that MAX MIN VERTEX COVER is FPT even when parameterized by the cardinality of a maximum matching $M$ of the input graph (recall that $m \leqslant \operatorname{opt}(G)$ ).

Proposition 5. MAX MIN VERTEX COVER can be optimally solved in time $O^{*}\left(3^{m}\right)$ where $M$ is maximum matching of the input graph.

Proof. Consider a general graph $G(V, E)$, and a maximum matching $M \subseteq E$ on $G$. All exposed vertices obviously form an independent set, that we denote by $S$. We also denote, as previously, by $V(M)$ the set of matched vertices. 
We show that any feasible solution $\operatorname{SOL}(G)$ for MAX MIN VERTEx COVER can be unequivocally characterized by its subset of matched vertices. Consider any subset $\operatorname{SOL}(G) \cap V(M)$ of $V(M)$ known to be the subset of a unknown feasible solution $\operatorname{SOL}(G)$. There actually exists a single solution $\operatorname{SOL}(G)$ which admits $\operatorname{SOL}(G) \cap V(M)$ as subset of matched vertices. Indeed denote by $\hat{S}$ the subset of $S$ containing all exposed vertices incident to a matched vertex that does not belong in $\operatorname{SOL}(G) \cap V(M)$. Then, the whole set $\hat{S}$ must be part of $\operatorname{SOL}(G)$ in order to make it feasible. Conversely, all exposed vertices that do not belong to $\hat{S}$ cannot belong to $\operatorname{SOL}(G)$, because they would make the solution non minimal: by definition, all of their neighbors already belong in the vertex cover.

Therefore, by identifying the subset OPT $\cap V(M)$, where OPT denotes a maximum minimal vertex cover, one would be able to reconstruct the whole solution OPT by simply adding to OPT $\cap V(M)$ all exposed vertices incident to a matched vertex not in the vertex cover.

Finally, notice that for each edge of the matching $M$, any vertex cover (a fortiori the optimal one) must take at least one endpoint of this edge. So, for each edge, any solution can take one endpoint, or the other, or both endpoints, that is three possibilities. Hence, there are at most $3^{m}$ vertex covers in the subgraph induced by the matched vertices.

Consider the following algorithm:

- compute a maximum matching $M$;

- build all $3^{m}$ possible vertex-covers $V_{i} \subseteq V(M)$ among the matched vertices;

- complete each of these vertex-covers by adding all vertices of $S$ incident to a vertex not in the vertex cover;

- output the maximal feasible solution.

It is clear that through the exhaustive search performed at step 2 of the algorithm, the subset OPT $\cap V(M)$ will be found, and when completed by exposed vertices at step 3 , the optimal solution will be produced. Hence, an optimal solution can be computed in $O^{*}\left(3^{m}\right)$, and the proof is concluded.

Taking into account that $m \leqslant \tau$, the cardinality of a minimum vertex cover of the input graph, the following corollary immediately holds.

Corollary 2. MAX MIN VERTEX COVER parameterized by $\tau$ is FPT.

Let us now quickly point out how combination of Propositions 4 and 5 allows us to handle interesting trade-offs between parameterization and approximation. Indeed, we shall show that approximation ratios for MAX MIN VERTEX COVER, impossible to achieve in polynomial time, can be achieved in parameterized time. This issue has been already studied in $[5,6,9,10,12]$, etc., for several problems, as Min verTEX COVER, SteINer TREe, Min EDGE DOMINATing SET, several restricted versions of MIN HITTING SET, etc.

Revisit Proposition 5 and remark that if $m<(\log 1.5874 / \log 3) \operatorname{opt}(G) \approx$ $0.42 \mathrm{opt}(G)$, then the parameterized algorithm of Proposition 5 runs faster than that of Proposition 4 while, if $m \geqslant 0.42 \mathrm{opt}(G)$, any minimal vertex cover (a fortiori the one of Proposition 3) achieves ratio greater than, or equal to, 0.42, ratio "forbidden" in polynomial time. For instance, we can guarantee ratio 0.1 
in parameterized time less than $O^{*}\left(1162^{\mathrm{opt}}\right)$, much smaller than $O^{*}\left(1.5874^{\mathrm{opt}}\right)$, or even, approximation ratio 0,4 in time less than $O^{*}\left(1.552^{\mathrm{opt}}\right)$ that always remains less than $O^{*}\left(1.5874^{\mathrm{opt}}\right)$.

We conclude the section by showing that the same kind of trade-off can me made combining Proposition 4 and Proposition 1 [4] in order to get approximation results unachievable in polynomial time through exponential algorithms running faster that the optimal ones. Recall that Proposition 1 in [4] claims that for any positive $\epsilon \leqslant 5$, MIN INDEPENDENT DOMINATING SET is $(1+\epsilon)$ approximable in time $O^{*}\left(1.3351^{(1-(\epsilon / 168)) n}\right)$.

If $\operatorname{opt}(G)<0.626(1-(\epsilon / 168)) n$, then the algorithm of Proposition 4 computes a maximum minimal vertex cover of $G$ in time smaller than $O^{*}\left(1.3351^{n}\right)$, which is the best worst-case complexity known for MIN INDEPENDENT DOMINATING SET and, consequently, for MAX MIN VERTEX COVER. Suppose now that $\operatorname{opt}(G) \geqslant 0.626(1-(\epsilon / 168)) n$. In this case the $(1+\epsilon)$-approximation algorithm of Proposition 1 in [4] (indeed this algorithm can be seen as a kind of moderately exponential approximation schema) can be transformed a moderately exponential approximation schema for MAX MIN VERTEX COVER.

Denote by $\operatorname{opt}^{\prime}(G)$ the size of a minimum dominating set in $G$ and use the algorithm of Proposition $1[4]$ in order to get an $(1+\epsilon)$-approximate independent dominating set $S$. Obviously, the set $C=V \backslash S$ is a minimal vertex cover of $G$. The approximation ratio of $C$ is:

$$
\frac{|C|}{\operatorname{opt}(G)}=\frac{n-|S|}{n-\operatorname{opt}^{\prime}(G)} \geqslant \frac{n-(1+\epsilon) \operatorname{opt}^{\prime}(G)}{n-\operatorname{opt}^{\prime}(G)}
$$

The last expression in (10) decreases with opt $^{\prime}(G)$; since opt $(G) \geqslant 0.626(1-$ $(\epsilon / 168)) n$, $\operatorname{opt}^{\prime}(G) \leqslant(0.374+(0.626 \epsilon / 168)) n$ and setting it in the last term of (10) we get after some easy but tedious algebra that $|C| / \operatorname{opt}(G) \geqslant 1+\epsilon^{\prime}$ for some $\epsilon^{\prime}$ that only depends on $\epsilon$.

\section{MAX MIN VERTEX COVER in interval graphs}

Recall that an interval graph $G(V, E)$ is defined as the intersection graph of a set of intervals on the real line. Each vertex $v_{i} \in V$ corresponds to an interval $l_{i}=$ $\left[x_{i}, y_{i}\right]$ of the interval set, and for each pair of vertices $\left(v_{i}, v_{j}\right)$, the edge $\left(v_{i}, v_{j}\right)$ is present in the graph if and only if intervals $l_{i}$ and $l_{j}$ intersect. Figure 1 provides an example of interval graph, and of the interval set associated.

Also recall that all interval graphs are chordal graphs, i.e., graphs where each cycle of four or more nodes has a chord, which is an edge joining two nodes that are not adjacent in the cycle.

WEIGHTED MIN INDEPENDENT DOMINATING SET and, consequently, WEIGHTED MAX MIN VERTEX COVER was shown to be solvable in linear time in chordal graphs with weights 0 or 1 [11]. However, it was proved in [18] that when weights are arbitrary, the problem becomes NP-hard and even inapproximable by any randomized polynomial-time algorithm within any ratio $c \ln (n)$ for some fixed constant $c$, unless $\mathbf{N P} \subseteq \mathbf{Z T I M E}\left(n^{O(\log \log n)}\right)$. 


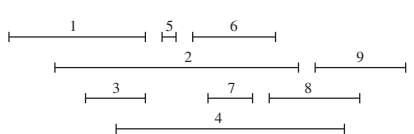

(a) The intervals ..

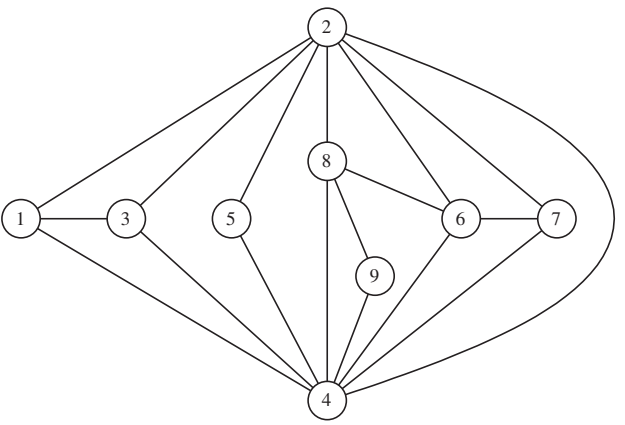

(b) $\ldots$ and the associated graph

Figure 1: An example of interval graph; for simplicity, in Figure 4 vertices have the same names as the corresponding intervals.

Here, we provide a positive result for WEIGHTED MAX MIN VERTEX COVER and WEIGHTED MIN INDEPENDENT DOMINATING SET in interval graphs when weights are arbitrary.

Proposition 6. Weighted MAX MIN Vertex COVER and Weighted MiN INDEPENDENT DOMINATING SET are solvable in polynomial time on interval graphs.

Proof. We develop an $O\left(n^{2}\right)$ dynamic programming algorithm that solves both problems. For simplicity, we analyze it for the case of WEIGHTED MIN INDEPENDENT DOMINATING SET.

Let $G(V, E, w)$ be a weighted interval graph, where each vertex $i$ is associated with a weight $w(i)$. We suppose that $G$ is connected; if not, it suffices to run the dynamic programming algorithm on each of the connected components. Suppose that vertices of $G$ are labelled in increasing order of $v_{i}$ from 1 to $n$. In a given set of intervals/vertices, the one with minimal index will be denoted as the first, while the one with maximal index will be referred as the last. Also, for each vertex vertex $v_{i}$, define $S\left(v_{i}\right)$ as a subset of vertices $j$ such that: (a) $j<i$, (b) $v_{j} \notin \Gamma\left(v_{i}\right)$ and (c) $\left\{v_{j+1}, \ldots, v_{i-1}\right\} \subseteq \Gamma\left(v_{j}\right) \cup \Gamma\left(v_{i}\right)$.

For example, in Figure 1, $S\left(v_{9}\right)=\left\{v_{2}, v_{6}, v_{7}\right\}$. Indeed, vertices $v_{4}$ and $v_{8}$ both belong to $\Gamma\left(v_{9}\right)$, while considering $j=1, j=3$ or $j=5$ induces that vertices $v_{6}$ and $v_{7}$ are not included in $\Gamma\left(v_{j}\right) \cup \Gamma\left(v_{9}\right)$ (as well as vertex $v_{5}$ for $j=1$ ).

Some slight modifications are performed on the graph before running the dynamic programming algorithm. Isolated vertices are simply deleted, knowing that such nodes cannot be part of any feasible solution. In order to simplify the initialization of the algorithm, we also add one vertex $v_{0}$, with $w\left(v_{0}\right)=0$, whose interval does not intersect with any other interval, and is placed in the very first position. This is done in order to ensure that the set $S\left(v_{i}\right)$ will not be empty for any $i \geqslant 1$. 
Finally, let $W_{i}$ denote the value of the minimum weight solution on the subgraph induced by vertices $v_{1}, \ldots, v_{i}$ such that vertex $v_{i}$ is a dominating vertex. Given the definition of $W_{i}$, it is clear that opt $=\min _{i \in\left\{v_{n}\right\} \cup \Gamma\left(v_{n}\right)}\left\{W_{i}\right\}$. Indeed, considering that intervals are labelled in increasing order of $i$, any interval $l_{i}$ that intersects $l_{n}$ also intersects all intervals $l_{i+1} \ldots l_{n-1}$, so that vertex $v_{i}$ dominates all vertices $v_{j}, j \leqslant i+1$, and a feasible solution on vertices $v_{1}, \ldots, v_{i}$ such that $v_{i}$ is dominating is feasible for the whole graph. On the other hand, if the last dominating vertex does not belong to $\left\{v_{n}\right\} \cup \Gamma\left(v_{n}\right)$, then $n$ is neither dominated nor dominating.

The dynamic programming is initialized with $W_{0}=0$, and the recursion formula is $W_{i}=w(i)+\min _{v_{j} \in S\left(v_{i}\right)}\left\{W_{j}\right\}, \forall i \geqslant 1$. Indeed, for any vertex $v_{i}$ that is included in the dominating set, then the last vertex $v_{j}$ before $v_{i}$ to be also dominating is necessarily in the set $S\left(v_{i}\right)$ :

- if $v_{j} \in \Gamma\left(v_{i}\right)$, then including $v_{j}$ and $v_{i}$ in the solution results in a nonindependent set;

- if $\exists k \in\{j+1, \ldots, i-1\}$ and $v_{k} \notin \Gamma\left(v_{j}\right) \cup \Gamma\left(v_{i}\right)$, then vertex $v_{k}$ will not be dominated; indeed, $v_{k}$ cannot be dominated by any vertex $v_{l}, l \geqslant i$, considering that vertices are labelled in increasing order of $i$ : if $i$ does not intersect $k$, then no following interval can do this; it cannot be dominated by any vertex $v_{l}, l \leqslant j$ either, considering that $W_{j}$ defines an independent dominating set: if a dominating interval $l<j$ intersects interval $k$, then it also intersects $j$, so that $W_{j}$ is not independent.

Therefore, if the value (and corresponding solution) $W_{j}$ is valid $\forall v_{j} \in S\left(v_{i}\right)$, then the value $W_{i}$ is valid itself. There are $n$ partial solutions $W_{i}$ to build, each of them needing $O(n)$ operations to be computed. So, the whole complexity is $O\left(n^{2}\right)$, as claimed.

\section{References}

[1] S. Arnborg. Efficient algorithms for combinatorial problems on graphs with bounded decomposability. a survey. BIT Numerical Mathematics, 25(1):1$23,1985$.

[2] S. Arnborg and A. Proskurowski. Linear time algorithms for NP-hard problems restricted to partial $k$-trees. Discrete Appl. Math., 23(1):11-24, 1989.

[3] H. L. Bodlaender. Dynamic programming on graphs with bounded treewidth. In T. Lepistö and A. Salomaa, eds, Proc. ICALP'88, LNCS 317, p. 105-118. Springer, 1988.

[4] N. Bourgeois, F. Della Croce, B. Escoffier, and V. Th. Paschos. Fast algorithms for min independent Dominating Set. Discrete Appl. Math., 161(4-5):558-572, 2013. 
[5] L. Cai and X. Huang. Fixed-parameter approximation: conceptual framework and approximability results. In H. L. Bodlaender and M. A. Langston, eds, Proc. IWPEC'06, LNCS 4169, p. 96-108. Springer, 2006.

[6] Y. Chen, M. Grohe, and M. Grüber. On parameterized approximability. In H. L. Bodlaender and M. A. Langston, eds, Proc. IWPEC'06, LNCS 4169, p. 109-120. Springer, 2006.

[7] M. Damian-Iordache and S. V. Pemmaraju. Hardness of approximating independent domination in circle graphs. In Proc. ISAAC'99, LNCS 1741, pages 56-69. Springer, 1999.

[8] R. G. Downey and M. R. Fellows. Parameterized complexity. Monographs in Computer Science. Springer, New York, 1999.

[9] R. G. Downey, M. R. Fellows, and C. McCartin. Parameterized approximation problems. In H. L. Bodlaender and M. A. Langston, eds, Proc. IWPEC'06, LNCS 4169, p. 121-129. Springer, 2006.

[10] B. Escoffier, J. Monnot, V. Th. Paschos, and M. Xiao. New results on polynomial inapproximability and fixed parameter approximability of edge dominating set. In D. M. Thilikos and G. J. Woeginger, editors, Proc. IPEC'12, LNCS 7535, p. 25-36. Springer, 2012.

[11] M. Farber. Independent domination in chordal graphs. Oper. Res. Lett., 1(4):134-138, 1982.

[12] M. R. Fellows, A. Kulik, F. A. Rosamond, and H. Shachnai. Parameterized approximation via fidelity preserving transformations. In A. Czumaj, K. Mehlhorn, A. Pitts, and R. Wattenhofer, editors, Proc. ICALP'12, LNCS 7391, p. 351-362. Springer, 2012.

[13] S. Gaspers, D. Kratsch, and M. Liedloff. Exponential time algorithms for the minimum dominating set problem on some graph classes. In L. Arge and R. Freivalds, editors, Proc. SWAT'06, LNCS 4059, p. 148-159. Springer, 2006 .

[14] S. Gaspers and M. Liedloff. A branch-and-reduce algorithm for finding a minimum independent dominating set in graphs. In F. V. Fomin, editor, Proc. $W G^{\prime} 06$ 4271, p. 78-89. Springer, 2006.

[15] M. M. Halldórsson. Approximating the minimum maximal independence number. Inform. Process. Lett., 46:169-172, 1993.

[16] C. Lund and M. Yannakakis. The approximation of maximum subgraph problems. In Proc. ICALP'93,LNCS 700, p. 40-51. Springer, 1993.

[17] M. Mucha and P. Sankowski. Maximum matchings via gaussian elimination. In Proc. FOCS'04, p. 248-255, 2004. 
[18] Y. Okamoto, T. Uno, and R. Uehara. Linear-time counting algorithms for independent sets in chordal graphs. In D. Kratsch, editor, Proc. WG'05, LNCS 3787, p. 433-444. Springer, 2005.

[19] J. M. M. van Rooij, H. L. Bodlaender, and P. Rossmanith. Dynamic programming on tree decompositions using generalised fast subset convolution. In F. Amos and S. Peter, editors, Proc. ESA'09, LNCS 5757, p. 566-577. Springer, 2009. 